\title{
An analysis of STIS HST UV spectra of M 33 early B supergiants ${ }^{\star}$
}

\author{
M. A. Urbaneja ${ }^{1}$, A. Herrero ${ }^{1,2}$, R. P. Kudritzki ${ }^{3}$, F. Bresolin ${ }^{3}$, L. J. Corral ${ }^{1}$, and J. Puls ${ }^{4}$ \\ 1 Instituto de Astrofísica de Canarias, 38200 La Laguna, Tenerife, Spain \\ 2 Departamento de Astrofísica, Universidad de La Laguna, Avda. Astrofísico Francisco Sánchez, s/n, \\ 38071 La Laguna, Spain \\ 3 Institute for Astronomy, University of Hawaii, 2680 Woodlawn Drive, Honolulu, Hawaii 96822, USA \\ 4 Universitäts-Sternwarte München, Scheinerstr. 1, 81679 München, Germany
}

Received 7 February 2002 / Accepted 4 March 2002

\begin{abstract}
We present terminal velocities of M 33 B-supergiants, obtained from STIS HST spectra as part of our programme to investigate the Wind Momentum - Luminosity Relationship (WLR) in the Local Group. Terminal velocities are derived from their N V, C IV, and Si IV resonance lines in UV spectra. Comparing with IUE spectra of Galactic B-supergiants we found evidence of low metallicity in three of our objects. The terminal velocities are consistent with the corresponding values of Galactic stars, except for B-133. For this star we find a very large $v_{\infty}$ and a red Si IV component deeper than the blue one, that might be an indication of binarity. The average ratio between terminal and turbulent wind velocities is 0.25 , well above the value found for Galactic stars.
\end{abstract}

Key words. stars: atmospheres - stars: early-types - stars: supergiants - stars: fundamental parameters - stars: winds, outflows - galaxies: individual: M 33

\section{Introduction}

The Wind Momentum-Luminosity Relationship (WLR, Kudritzki et al. 1995; Puls et al. 1996, for recent reviews see Kudritzki 1998 and Kudritzki \& Puls 2000) offers the very attractive opportunity to derive the stellar luminosity directly from the analysis of the observed spectrum, provided that the wind is radiatively driven. Accurate individual stellar distances can then be obtained if the apparent magnitude and extinction are known. This method, that has been extensively described elsewhere (see for example the reviews cited above), can be potentially applied to individual stars up to distances of $10-20 \mathrm{Mpc}$, reaching the Virgo and Fornax clusters with accuracies as low as $0.1 \mathrm{mag}$, using present day telescopes and techniques (McCarthy et al. 1997; Kudritzki et al. 1999).

However, accurate application of the method requires previous calibrating work, as the WLR depends on the stellar metallicity and the spectral type (Puls et al. 1996; Kudritzki et al. 1999). Thus observation and analysis of stars of different spectral type and metallicity at known distances are crucial to calibrate the method before applying it.

Send offprint requests to: M. A. Urbaneja,

e-mail: maup@ll.iac.es

* Partly based on INES data from the IUE satellite.
For this reason we have started in our group a number of programs aimed at calibrating the WLR for different stars in different environments within the Local Group (Puls et al. 1996; McCarthy et al. 1997; Kudritzki et al. 1999; Herrero et al. 2001).

In the present paper, we analyze STIS HST UV spectra of M $33 \mathrm{~B}$-supergiants. Our analysis is primarily aimed at obtaining the wind terminal velocities, needed to calculate the WLR, that will be determined after the analysis of the optical spectrum.

In addition our study constitutes, together with a similar study of B-supergiants in M 31 (Bresolin et al. 2002), the largest homogeneous sample of stars analyzed in the UV beyond the Milky Way and the Magellanic Clouds (see Prinja \& Crowther 1998, for a recent list), and an important increase in the total number of analyzed Bsupergiants, in particular those with large intrinsic brightness (bright magnitudes are of course still a primary selection criteria for our extragalactic targets). The group of early B-supergiants is particularly important for the spectral type dependence of the WLR. Between O9 and B3, two jumps are observed in the WLR, one between O9 and B0 and another between B1 and B1.5, while the WLR for B-supergiants between B1.5 and B3 is not well understood in terms of the radiatively driven wind theory, as wind momenta lower than expected are derived for the analyzed Galactic stars (Kudritzki et al. 1999). 
Table 1. M 33 stars observed with HST/STIS. The first column gives the star identification in the catalog of Ivanov et al. (1993) (IFM93), while the second one gives the identification by Humphreys \& Sandage (1980) (HS80). Coordinates are taken from Ivanov et al. (1993) and precessed for equinox 2000, except for B-38 and B-133, for which coordinates from Massey et al. (1996) are taken. Magnitudes are from the same sources, while spectral types are from Monteverde et al. (1996) and Monteverde et al. (2000), except for 0900 and 1137, for which new spectral types are given (that will be justified in a forthcoming paper).

\begin{tabular}{rrccrlrlr}
\hline \hline $\begin{array}{r}\text { Ident } \\
\text { IFM93 }\end{array}$ & $\begin{array}{r}\text { Ident } \\
\text { HS80 }\end{array}$ & $\alpha(2000)$ & $\delta(2000)$ & $\begin{array}{r}V \\
\text { mag }\end{array}$ & $\begin{array}{l}\text { Spectral } \\
\text { Type }\end{array}$ & $\begin{array}{r}\text { Obs. } \\
\text { Date }\end{array}$ & $\begin{array}{l}\text { Exp. } \\
\text { time(s) }\end{array}$ & $S / N$ \\
\hline 0900 & & 013344.7 & 303618 & 17.3 & B0-B1 I & Jul.-8-00 & 5193 & 24 \\
0785 & 110-A & 013341.0 & 302237 & 16.1 & B1 Ia+ & Aug.-7-99 & 5201 & 29 \\
& B-38 & 013300.8 & 303505 & 16.7 & B1 Ia & Sep.-5-00 & 5205 & 30 \\
0515 & B-133 & 013329.0 & 304744 & 17.6 & B1.5 Ia & Aug.-5-99 & 5205 & 30 \\
1733 & B-526 & 013415.9 & 303346 & 17.6 & B2.5 I & Dec.-11-00 & 5201 & 15 \\
1137 & & 013353.1 & 303528 & 16.7 & B3 I & Jul.-9-00 & 5205 & 15 \\
\hline
\end{tabular}

In Sect. 2 we show the observations of the spectra, that are described in Sect. 3. Section 4 presents the analysis of the UV profiles, while in Sect. 5 we discuss the individual aspects found for each star. In Sect. 6 we present the discussion of the results and the conclusions.

\section{The observations}

Observations were made with the HST STIS, using the GL140 grating which provides a resolution from 310 to $210 \mathrm{~km} \mathrm{~s}^{-1}$ in the wavelength range from 1150 to $1700 \AA$. We used a 0.2 wide slit, which is recommended for optimizing the spectral purity. Table 1 gives our list of objects and other details of the observations.

There are two spectra for each star. We merged these two spectra after checking their relative displacement by cross-correlating them.

Merged stellar spectra are corrected for the relative velocity between stars and observer. This is done by measuring the displacement from rest wavelength of metal lines that are purely photospheric. There are a few photospheric lines that could in principle be used in the observed spectral range (Prinja 1990). They are difficult to identify in all spectra. Thus, after inspection of the spectra, we decided to use the Si III doublet at $\lambda \lambda 1500.24,1501.19 \AA$, because it is the only one that can be clearly identified in all spectra. The spectral resolution does not allow us to resolve both components of the Si III doublet. We use as rest wavelength that of a composed line centered at $\lambda 1500.72 \AA$. Because of the small signal to noise ratio of the spectra, we decided to correct one of the spectra of higher quality and then refer the others to this one. We selected the spectrum of M 33-0900 for that purpose. Once the M 33-0900 spectrum had been shifted so that the Si III line was at rest wavelentgh, we checked this correction with the position of other strong interstellar lines (see f.e. Prinja 1990), as these are probably produced by the interstellar medium close to M 33-0900. We can see in Fig. 1 that the shifted interstellar lines are at their expected rest wavelengths.

To correct the other spectra we cross-correlated them with the corrected spectrum of M 33-0900. To avoid possible biases, strong P-Cygni profiles have been masked ${ }^{1}$ before correlations (see Howarth et al. 1997). For M 331137 and M 33-B-526, the stars with lowest SN spectrum, the cross-correlation function does not present a well defined maximum. Checking the strong interstellar lines present in the spectra (Si II $\lambda \lambda 1260.40,1526.70)$ we found good concordance between all spectra, excluding M 33-1137 and M 33-B-526. We decided to shift both spectra until the position of their interstellar lines agree with those of other spectra. Special care was taken in the case of M 33-B-526, as the unshifted spectrum shows several lines in the vicinity of the rest wavelength of the intestellar lines.

After having shifted the spectra we rectified the continuum by tracing a polynomial through a number of selected continuum points chosen iteratively, in the same way as described in Herrero et al. (2001).

\section{Description of the spectra}

After all the above corrections have been performed, we finally obtained the spectra displayed in Fig. 1.

One of the difficulties of stellar analyses in M 33 is the large possible variation in metallicity, that complicates the interpretation of the spectrum. Thus in Fig. 2 we show a comparison of the N V, Si IV and C IV lines of the M 33 stars with Galactic stars of similar spectral classification. Their identifications and spectral types are given in Table 2.

The Galactic UV spectra have been taken from the INES IUE archive, and have been degraded from the original high resolution to the lower resolution of our M 33 HST spectra. We emphasize that we have taken the rectification points of the M 33 stars as a guide to rectify the IUE spectra in the same way. Therefore, the extra absorption seen redwards of the Si IV and CIV profiles in some Galactic stars are probably an effect of metallicity. Furthermore these extra absorptions are consistent with the profiles indicating a lower metallicity in the M 33 stars. The $\mathrm{N} v$ comparison is strongly affected by the different L $\alpha$ absorptions.

\footnotetext{
${ }^{1}$ Spectral regions of $\mathrm{N} v, \mathrm{C}$ IV, Si IV UV resonance lines have been set to zero.
} 


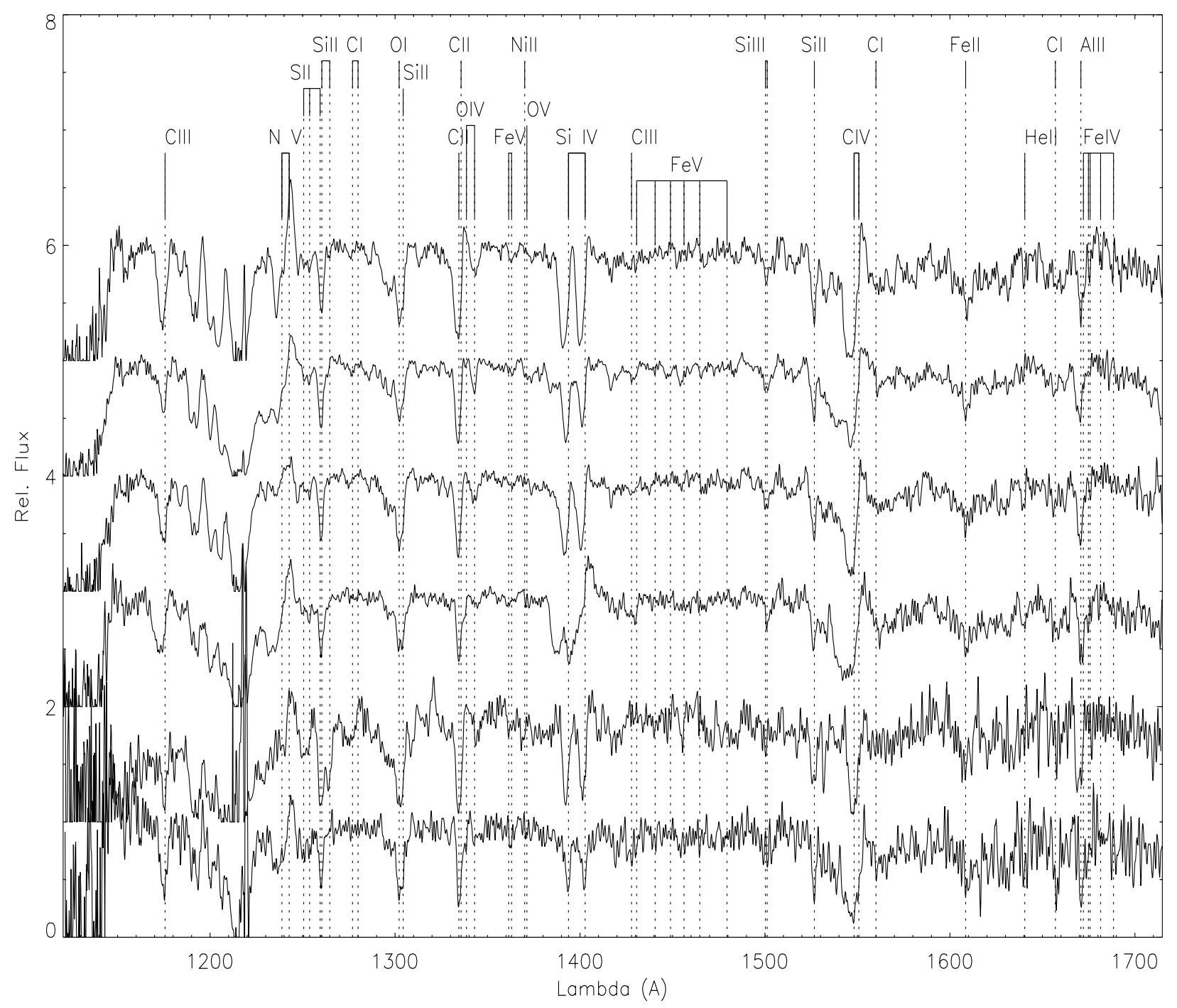

Fig. 1. The observed rectified UV spectra. From top to bottom: M 33-0900, M 33-110-A, M 33-B-38, M 33-B-133, M 33-B-526 and M 33-1137. Relative fluxes have been arbitrarily displaced in ordinates for the sake of clarity. The main IS lines are marked at the top, and below we have indicated the rest wavelengths of the most important stellar lines.

M 33-0900 has Si IV and CIV profiles that are very similar to those of HD 154090. Although Nv is stronger in M 33-0900, the star looks normal for its spectral type.

This is not the case for $\mathbf{M} \mathbf{3 3 - 1 1 0 - A}$, which in comparison to HD 148688 displays very weak $\mathrm{Si}$ and C lines. Moreover considering the luminosity effect we would expect for Si IV, the difference is even larger. This is consistent with the low $\mathrm{Si}$ and $\mathrm{O}$ abundances derived by Monteverde et al. (2000). Thus we can expect a low metallicity for 110-A, also consistent with the higher continuum level redwards from Si IV and Civ. The Nv profile is again stronger than in the comparison star. The slopes of the blue wings of both the $\mathrm{Si}$ and $\mathrm{C}$ lines are smaller than in HD 148688. This will be interpreted during the fit procedure as a larger dispersion velocity. Finally we shall mention the broad absorption in the C IV blue wing that becomes more apparent due to the low absorption in this line. This additional absorption is present in both individual M 33-110-A spectra and can be clearly indentified in Fig. 3 where we show a comparison of the C IV line in 110-A and in B-38. We have used this figure as a guide in fitting the $\mathrm{C}$ IV line in 110-A.

M 33-B-38, for which Monteverde et al. (2000) again derive low $\mathrm{O}$ and $\mathrm{Si}$ abundances, has also been compared with HD 148688. Similar comments as for 110-A are applicable, although effects are less extreme and the $\mathrm{N} v$ profile in this case is marginal (as in the Galactic star).

This has previously been observed and analized by Bianchi et al. (1996) and was later reanalyzed by Prinja \& Crowther (1998). Both used the same HST GHRS spectrum, which looks like ours, including the lower slope in the blue wings as compared to Galactic stars (see Fig. 4 from Bianchi et al. 1996). Bianchi et al. (1996) find a narrow absorption feature (narrow absorption component, NAC) at a blueshifted velocity of $\sim 1250 \mathrm{~km} \mathrm{~s}^{-1}$, which is close to the edge velocity determined by 

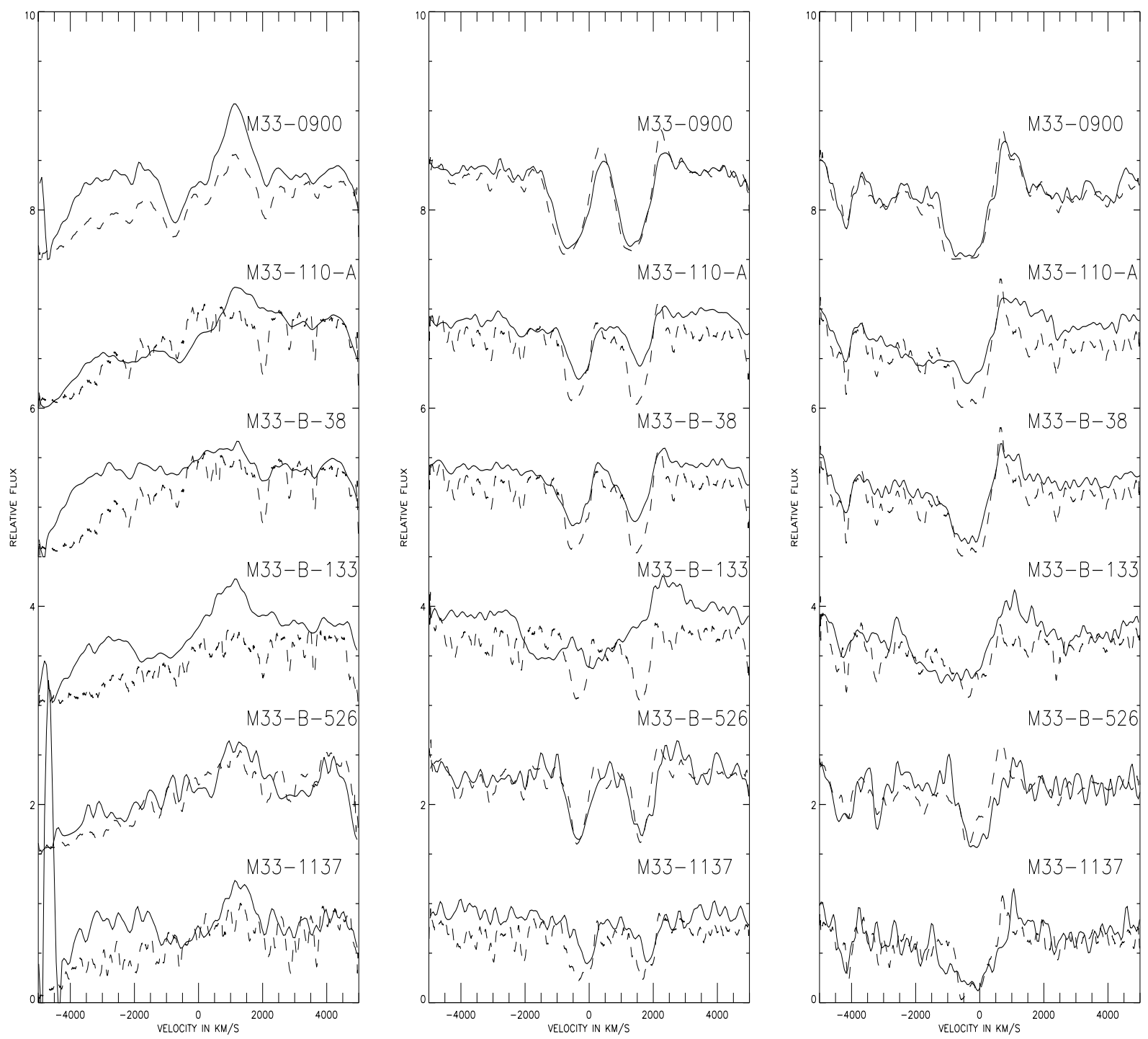

Fig. 2. Comparison of the Nv (left), Si IV (middle) and C IV (right) lines in the M 33 (full line) and Galactic (dashed line) stars. From top to bottom: M 33-0900 and HD 154090; M 33-110-A and HD 148688; M 33-B-38 and HD 148688; M 33-B-133 and HD 38711; M 33-B-526 and HD 198487; M 33-1137 and HD 51309. Spectral clssifications are given in Table 2. Relative fluxes have been arbitrarily displaced in ordinates for the sake of clarity. Galactic spectra have been degraded to the M 33 HST resolution.

Prinja \& Crowther (1998), $1225 \mathrm{~km} \mathrm{~s}^{-1}$. Interestingly, we also find evidence of a NAC at the same velocity, which may indicate that this is a more permanent feature.

M 33-B-133 shows the most puzzling spectra. The red component of the Si IV doublet is deeper than the blue one, which cannot be explained by the theory. The effect is due neither to noise nor to variability, as it is present in the same form in both individual spectra of M 33-B-133. We see numerous features in the C IV profile that could be interpreted as NACs. From them, only the absorptions at 250, 585 and $1990 \mathrm{~km} \mathrm{~s}^{-1}$ show both components. None of these NACs, however, seems strong enough to explain the red component of Si IV.

This is not the only remarkable feature in the spectrum of M 33-B-133. This object has been classified by
Monteverde et al. (2000) as B1.5 Ia, and is compared with HD 152236 (B1.5 Ia+) in Fig. 2. We see that the profiles are quite different. In fact, the spectrum of B-133 resembles more that of $\kappa$ Ori (HD 38771), a B0.5 Ia star, although a comparison of the optical spectra excludes such an early spectral type for M 33-B-133.

Another possibility is a blend with the spectrum of a hotter star. This is supported by the resemblance of the spectra of B-133 and $\kappa$ Ori, and by the data in Table 1 . Note that there B-133 has a larger $S / N$ than would be expected from a comparison of its visual magnitude and spectral type with those of the other stars. Thus, although a particular set of circumstances is required because the B1.5 supergiant dominates the blue spectrum, it is not impossible that a hotter companion of type O9.5-B0.5 is 


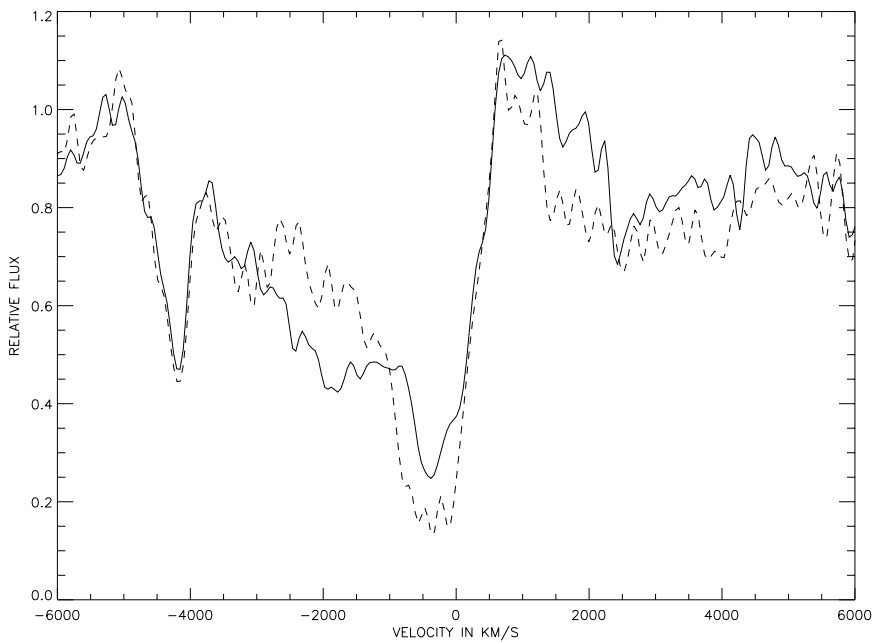

Fig. 3. Comparison of the C IV line in M 33-110-A (solid line) and M 33-B-38 (dashed line).

dominating the UV spectrum. If this is the case the apparent strong red component of the Si IV doublet would be the main contribution of the B1.5 star in the UV spectrum.

However, we could not reproduce the observed spectrum by combining IUE spectra of different spectral types without extra ad hoc assumptions (as arbitrary relative displacements), nor can we detect any clear indication of binarity in the HST WFPC2 images of B-133, or other features indicative of a companion in the blue and UV spectrum, and thus the suspected binary nature of B-133 remains unconfirmed.

M 33-B-526 displays lines very similar to those of HD 198487, in spite of its suspected binary nature from its appearance in the Keck I screen (McCarthy, private communication).

The C IV profile displays several absorption features redwards of the rest wavelength that could be interpreted as contributions from a companion star. However, their positions do not correspond to those of the doublet separation Thus we expect this to be the spectrum of a single star.

M 33-1137 has Si IV profiles that are clearly weaker than those of the selected Galactic counterpart, HD 51309. The CIV profile in M 33-1137 is clearly broader than in HD 51309, with a shallow slope in the blue wing. It displays additional absorptions that agree with the positions of the $\mathrm{C}$ IV components at $-75 \mathrm{~km} \mathrm{~s}^{-1}$, thus clearly pointing to wind inhomogeneities. Weaker absorptions are compatible with components at $-550 \mathrm{~km} \mathrm{~s}^{-1}$. Other absorptions cannot be clearly assigned to the C IV doublet, specially considering the low SNR. The N v feature is more evident in M 33-1137 than in the Galactic star. The observed spectrum points again to a lower metallicity in the M 33 star than in the Galactic comparison star.

Therefore the UV spectral morphology of the observed M 33 stars separates them in three groups: a group (particularly evident in Si IV) with lines weaker than their Galactic comparison stars (M 33-110-A, M 33-B-38 and
M 33-1137), a second group with lines similar to their Galactic counterparts (M 33-0900 and M 33-B-526) and one star (M 33-B-133) showing peculiar profiles for its spectral type. In addition, the M 33 stars seem to have lower slopes in the blue wings, pointing to larger dispersion velocities. This is particularly clear in the first group of stars.

\section{Analysis of the resonance lines}

We use the method described by Haser (Haser 1995, see also Lamers et al. 1999) to analyse the UV P Cygni lines in order to derive wind terminal velocities. We have also derived H I column densities towards the M 33 stars by fitting the IS L $\alpha$ line, in the same way as in Herrero et al. (2001) (see also Jenkins 1970; Bohlin 1975). The values quoted in Table 2 for these column densities are similar to those obtained for stars in our Galaxy (see Fig. 2 of Shull \& Van Steenberg 1985).

After continuum rectification, we extract small regions of each spectrum centered in our strategics doublets: N v $\lambda \lambda$ 1238.819, 1242.798; Si IV $\lambda \lambda$ 1393.73, 1402.73 and C IV $\lambda \lambda 1548.191,1550.761$. In case of discrepancies or difficulties, we give more weight to the Si IV and C IV lines than to the Nv one.

To get the wind terminal velocities we have to correct for the underlying photospheric components, which we do in an approximate way, by using IUE spectra of hot stars with weak winds (and projected rotational velocities as low as possible) as templates. These templates have been convolved with appropriate rotational profiles, to account for the individual stellar rotational speeds. We excluded $\mathrm{N} \mathrm{V}$ from this procedure, as the corresponding spectra are noisy and do not show any features.

We selected a sample of Milky Way dwarfs with spectral types from $\mathrm{O} 9 \mathrm{~V}$ to B3V taken from the INES database. This covers the spectral type range of our M 33 stars. Selection of photospheric template and continuum rectification has a big impact when fitting emission peaks, but has little effect on the determined terminal velocities. The IUE spectra have been corrected for the relative velocity between stars and observer, in the same way as for M 33 stars. The stars used for the photospheric templates are specified in Table 2. Note that the profiles of interest are very weak in these stars, but even so the different metallicity may be a small source of error.

The velocity stratification is parameterized as a usual $\beta$-law, and we account for the wind turbulent velocity in the same manner as described in (Haser 1995) and Herrero et al. (2001). The indetermination in the exponent of the velocity field, $\beta$, produces an additional uncertainty in the terminal velocities.

Table 2 gives the fit results with respect to the wind and turbulent velocities. The final fit to each line is shown in Figs. 4 and 5. 

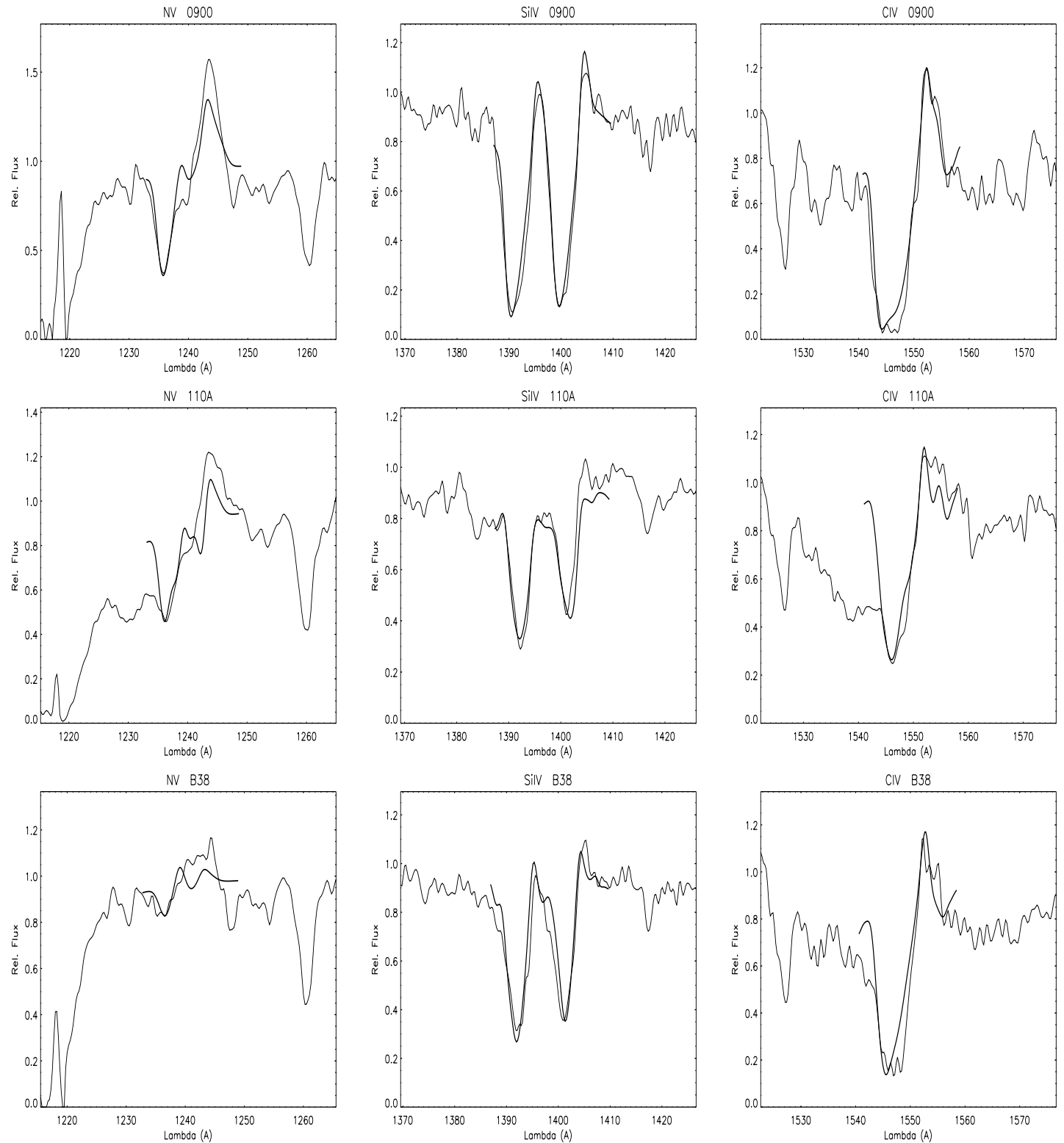

Fig. 4. Final fits for the three earliest stars of our sample. Lines are plotted from left to right (N v, Si IV, C IV) and stars from top to bottom in the same order as they are listed in the tables.

\section{Individual comments}

We briefly comment here on individual aspects of the analysis that could be of interest.

\subsection{33-0900}

We used HD 22591 (B0.5 V) for the photospheric profiles. The N V profile shows a large emission peak that we cannot reproduce completely (see Sect. 3 ). The $v_{\mathrm{ta}} / v_{\infty}$ ratio is 0.18 , larger than in Galactic stars.

\section{2. $M$ 33-110-A}

We used HD 39777 (B1.5 V) for the underlying photospheric profiles. We find the same problems as for M 33-0900: a large red emission peak in N v, and a large ratio $v_{\mathrm{ta}} / v_{\infty}$, which with a value of 0.25 is much larger than in Galactic B-supergiants. Furthermore, the additional absorption contaminating the blue side of CIV makes the fit a bit more difficult, affecting mainly the determination of $v_{\mathrm{ta}}$. This does not seriously affect the uncertainty in the adopted values for $\beta, v_{\infty}$ and $v_{\mathrm{ta}}$.

\section{3. $M$ 33-B-38}

The terminal velocity of this star has also been determined by Bianchi et al. (1996) and Prinja \& Crowther (1998). The first authors use an analysis method similar to the one employed here, and thus it is not surprising that our value agrees with their. Prinja \& Crowther (1998) determine the terminal velocities of their stellar sample from the violet edges of the profiles and the NACs. 

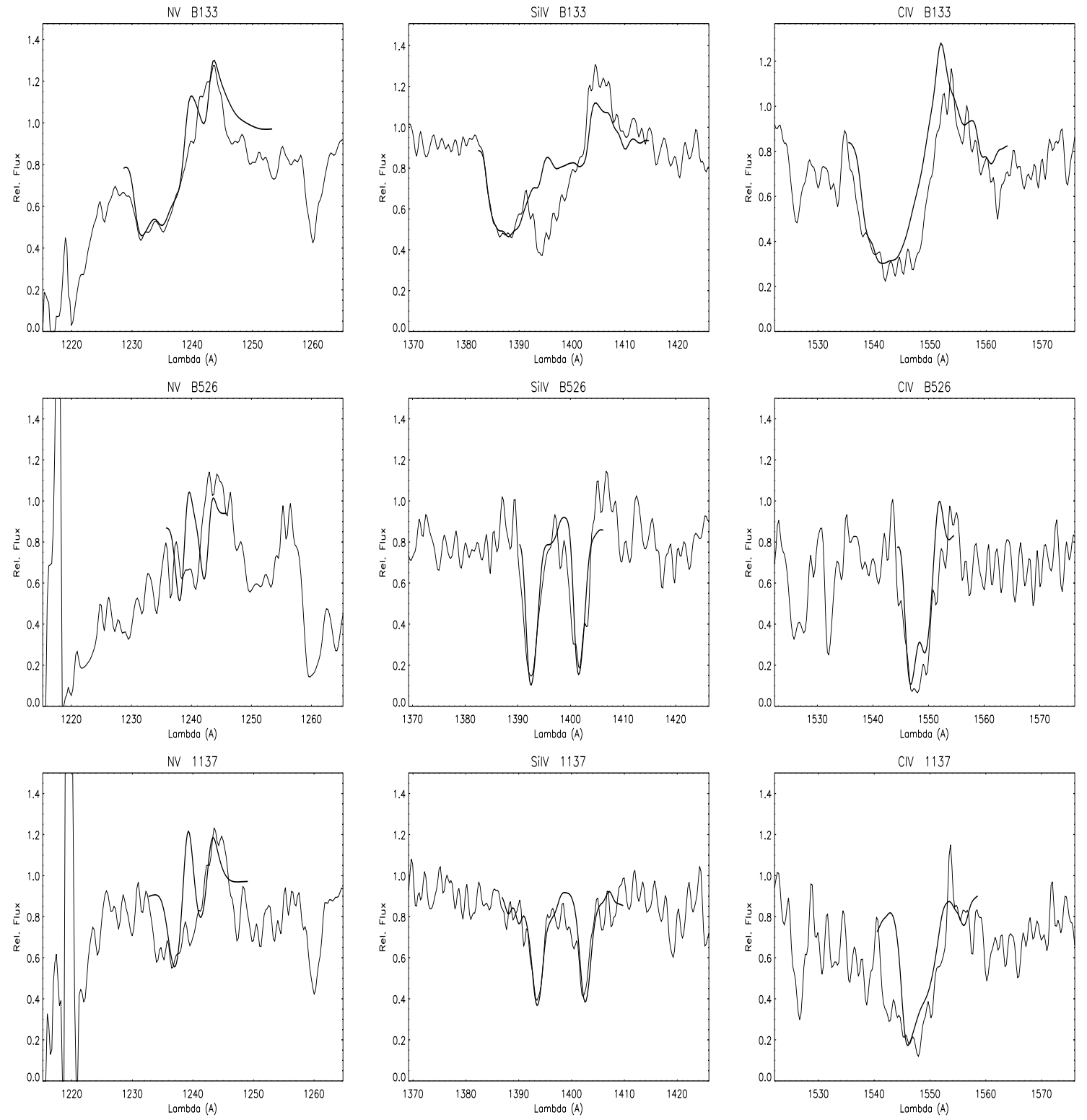

Fig. 5. As Fig. 4, however for the three last stars in Table 1.

The velocity they obtain for this star is much larger $\left(1225 \mathrm{~km} \mathrm{~s}^{-1}\right)$ than ours $\left(730 \mathrm{~km} \mathrm{~s}^{-1}\right)$ or the one by Bianchi et al. (about $700 \mathrm{~km} \mathrm{~s}^{-1}$ ). Our velocity would support the interpretation that what we have seen at $1250 \mathrm{~km} \mathrm{~s}^{-1}$ is actually not a NAC.

Bianchi et al. (1996) do not give the turbulent velocity of their stars. We derive a very large value, $250 \mathrm{~km} \mathrm{~s}^{-1}$, nearly $35 \%$ of $v_{\infty}$.

We adopted a large $\beta$-value, both to improve the consistency between the Si IV and C IV fits, and to improve the fit of the blue side of the red emission peak.

\section{4. $M$ 33-B-133}

We have used HD 39777 for phostospheric profiles, as in the case of M 33-110-A and M 33-B-38.
In spite of the low $\mathrm{O}$ and $\mathrm{Si}$ abundances derived by Monteverde et al. (2000) for this star, its UV spectrum shows comparatively strong C IV and Si IV P-Cygni profiles, indicating a strong and fast wind (see Sect. 3). The terminal velocity reaches $2050 \mathrm{~km} \mathrm{~s}^{-1}$. The turbulent velocity is $150 \mathrm{~km} \mathrm{~s}^{-1}$, a modest $7 \%$. This is the only star for which we obtain a terminal velocity clearly above the galactic average for its spectral type (taken from Kudritzki $\&$ Puls 2000), thus challenging the low abundances or the spectral classification (or both!). The spectral type, however, should change from B1.5 to O9 in order to have a terminal velocity close to the spectral type average. This is completely ruled out from inspection of the optical spectrum.

The fits displayed in Fig. 5 also show a behaviour different from those of the other stars. $\mathrm{NV}$ is well fitted. 
Table 2. Results obtained for the observed stars. $v_{\infty}$ and $v_{\text {ta }}$ (turbulence velocity in outer wind) and the uncertainty of their sum, $\Delta v$, are given in $\mathrm{km} \mathrm{s}^{-1}$.

\begin{tabular}{rlllcccrc}
\hline \hline Ident & $\begin{array}{l}\text { Spectral } \\
\text { Type }\end{array}$ & $\begin{array}{l}\text { Galactic } \\
\text { Star }\end{array}$ & $\begin{array}{l}\text { Spectral } \\
\text { Type }\end{array}$ & $\begin{array}{c}\text { log } \\
N(\mathrm{H} \mathrm{I})\end{array}$ & $v_{\infty}$ & $v_{\text {ta }}$ & $\Delta v$ & $\beta$ \\
\hline M 33-0900 & B0-B1 I & HD 154090 & B0.7 Ia & 20.85 & 950 & 170 & 50 & 1.0 \\
M 33-110-A & B1 Ia+ & HD 148688 & B1 Ia & 21.15 & 800 & 200 & 50 & 1.0 \\
M 33-B-38 & B1 Ia & HD 148688 & B1 Ia & 20.70 & 730 & 250 & 50 & 2.0 \\
M 33-B-133 & B1.5 Ia & HD 152236 & B1.5 Ia+ & 20.97 & 2050 & 150 & 100 & 1.0 \\
M 33-B-526 & B2.5 I & HD 198487 & B2.5Ia & 21.10 & 380 & 120 & 75 & 2.0 \\
M 33-B-1137 & B3 Ia & HD 51309 & B3Ib & 20.85 & 750 & 250 & 100 & 2.0 \\
\hline
\end{tabular}

The fit of the C IV profile is good in the bluest part of the wind absorption profile but is bad in the rest of the profile. Fortunately, the first one is the important part for determining the terminal velocity. The unsatisfactory fit at low wind velocities is cosmetically very dependent on the underlying photospheric profile, and thus does not mean very much by itself, but it would be consistent with the presence of additional C IV absorption at low wind velocities. We keep the low $\beta$ value as there is no need to increase $\beta$ to improve the consistency between C IV and Si IV. However, the anomaly in Si IV indicated in the preceding section, i.e., a stronger red component, can also not be fitted and indicates an extra absorption at low wind velocities. This uncertainty does not affect the determination of the terminal and turbulent wind velocities, that have an accuracy of $\pm 100 \mathrm{~km} \mathrm{~s}^{-1}$.

This does not solve the problem referred to above. The star has a large $v_{\infty}$ for its spectral classification and a Si IV red component stronger than the blue one. The first might be attributed to a case of bi-stability (Pauldrach \& Puls 1990), similar to that of P-Cygni in our Galaxy, where the ratio of terminal velocity to escape velocity is larger. This however does not explain the red Si IV component. One possible solution to this puzzle is that we are looking at a composite spectrum, B-133 being the star contributing to the red component of Si IV (see Sect. 3). If this is the case, we estimate its terminal wind velocity to be of the order of $450 \mathrm{~km} \mathrm{~s}^{-1}$, but would expect to see extra absorption in Si IV at low wind velocities.

\section{5. $M 33-B-526$}

It has been classified as B2.5I (Monteverde et al. 1996), from its optical spectrum. We used a B2.5V galactic star for the photospheric profiles (HD 44402, Z CMa).

The derived terminal velocity is the lowest in our sample and the UV spectrum shows only weak signs of mass loss. However, the turbulence velocity is relatively high, with a ratio $v_{\text {ta }} / v_{\infty}$ in excess of 0.3 . This large value is the result of a compromise between the fit of C IV and that of SiIV. The first would allow a lower turbulence, but then we cannot fit the second one. Again, the value of $\beta$ has to be large to favour consistency between both doublets and a better fit to C IV. However, the terminal velocity is not seriously affected. The uncertainty of both velocities is different now, being that of $v_{\mathrm{ta}} \pm 100 \mathrm{~km} \mathrm{~s}^{-1}$

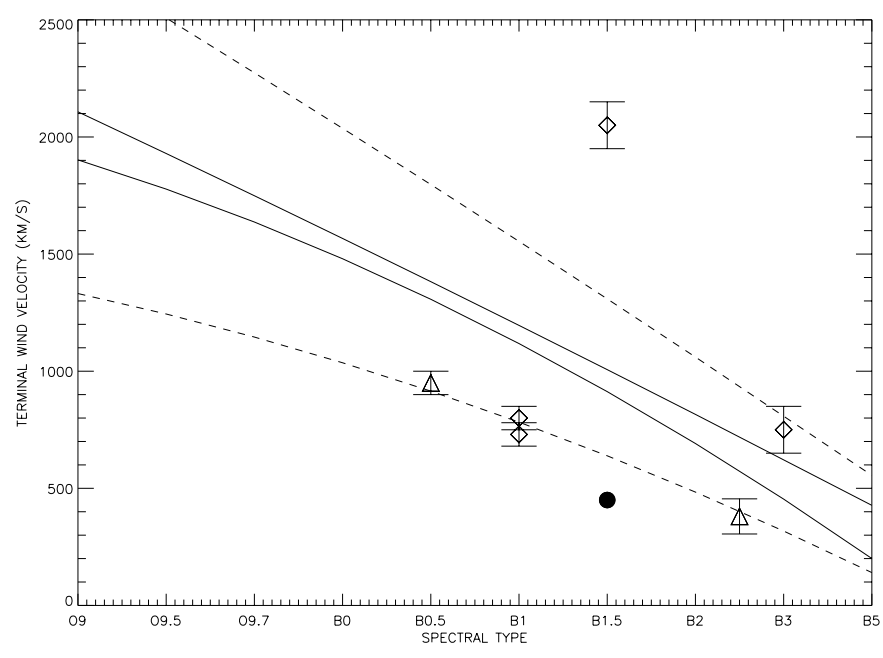

Fig. 6. The $v_{\infty}$ obtained for the M 33 stars. Solid lines represent the fits to OI + BIa (lower curve) and to OII + BIb supergiants (upper curve) of the Kudritzki \& Puls (2000) Galactic data. Dashed lines are $\pm 30 \%$ variations of these curves. The solid dot indicates the approximate position of B-133 if derive it from fitting the red Si IV component (see text for details).

and that of $v_{\infty} \pm 50 \mathrm{~km} \mathrm{~s}^{-1}$. For the sum of both we have adopted $\pm 75 \mathrm{~km} \mathrm{~s}^{-1}$.

\section{6. $M$ 33-1137}

This is the coolest star of our sample, with spectral type B3Ia. We take HD 32630 (B3V) for the photospheric profiles.

The Si IV doublet is mainly photospheric or in any case the wind has a small contribution. The fit to $\mathrm{N} \mathrm{V}$ is poor.

The C IV is the main profile for deriving the parameters of the velocity field. The low SNR of the spectra and the additional absorptions described in the preceding section make the fit difficult. We find the best fit at $750 \mathrm{~km} \mathrm{~s}^{-1}$, $250 \mathrm{~km} \mathrm{~s}^{-1}$ and 2.0 for $v_{\infty}, v_{\mathrm{ta}}$ and $\beta$. Again, $v_{\infty}$ is larger than the Galactic average from Kudritzki \& Puls (2000), but now much more moderately than for B-133. The uncertainties are slightly larger than those of the other fits, as already expected from the described difficulties. We adopt $\pm 100 \mathrm{kms}^{-1}$ for $v_{\infty}, v_{\mathrm{ta}}$ and its sum. 


\section{Discussion and conclusions}

In Fig. 6 we have plotted the derived $v_{\infty}$ against the stellar spectral types, together with parabolic fits to the average values quoted by Kudritzki \& Puls (2000) for Galactic OB supergiants. The fits have been obtained by joining OI and BIa supergiants on the one hand and OII and BIb supergiants on the other. We have also plotted lines that indicate a $30 \%$ variation from the plotted average relations, a usual scatter range (see Fig. 4 in Kudritzki \& Puls 2000).

All M 33 B-supergiants give us values that can be considered normal, except B-133. We see no difference between the stars with different suspected metallicities. This is in agreement with Puls et al. (2000) (see also Vink et al. 2000) who have argued that the terminal velocity (which depends on the slope of the line-strength distribution function, $\alpha$ ) is primarily controlled by the ratio of light ions vs. iron group elements, because of the different line statistics. As long as this ratio is roughly similar, the theoretical expected change of terminal velocity (due to the "indirect" $\alpha$ effect, see Puls et al. 2000, Sect. 5.2) is much smaller than if this ratio would be changed. In particular if a dense wind is present (as is the case for our supergiants) and this ratio remains unchanged (which we have to assume for the moment), the effect is expected to be very small (see Puls et al. 2000, Fig. 27), since the effective $\alpha$ then remains roughly constant. Only for thin winds and/or a significantly lower (general) metallicity the effect should become observable.

We could not find a satisfactory explanation for B-133. While its terminal velocity is well above the average for its spectral type, it could still be accepted (even with the low metallicity derived by Monteverde et al. 2000) assuming for example a bi-stability phenomenon, were it not for the anomalous red component of the Si IV doublet. Assuming on the other hand that B-133 is actually producing the red Si IV component, its velocity would be much closer to the average of its spectral type. However, we could not reproduce the observed profiles without ad hoc hypothesis, nor find conclusive evidence of a binary nature.

Although three of our stars have been analyzed by Monteverde et al. (2000) we will postpone a discussion of the terminal velocities in terms of the stellar parameters, as new analyses of their optical spectra, now including mass-loss effects, are currently under way in our group together with a set of newly observed stars.

The wind turbulent velocities derived here are larger than the typical $10 \% v_{\infty}$ (Kudritzki \& Puls 2000) or the $14 \%$ found by Herrero et al. (2001), reaching nearly $35 \%$ in the most extreme case. Including B-133 we obtain 0.25 for the mean $v_{\mathrm{ta}} / v_{\infty}$ ratio. This result is of the same order of that found by Bresolin et al. (2002) in their analysis of M 31 B supergiants. We tentatively attribute it for the moment to the extreme character of our objects, selected among the brightest supergiants in M 33, and as a consequence are among the most luminous objects.
Finally, we have detected evidence of numerous NACs, confirming the wide presence of wind inhomogenities.

Acknowledgements. We would like to thank F. Najarro for very useful discussions during the stellar analyses. A.H. wants to acknowledge support for this work by the spanish DGI under proyect AYA2001-0436, the DGES under project PB97-1438C02-01 and from the Gobierno Autonómico de Canarias under project PI1999/008. F.B. and J.P. acknowledge support from the German DLR, under grant RD-RX 50OR9909/2.

\section{References}

Bianchi, L., Hutchings, J. B., \& Massey, P. 1996, ApJ, 111, 2303

Bohlin, R. C. 1975, ApJ, 200, 402

Bresolin, F., Kudritzki, R. P., Lennon, et al. 2002, ApJ, in preparation

Haser, S. M. 1995, Ph.D. Thesis, Universitäts-Sternwarte der Ludwig-Maximillian Universität, Munich

Herrero, A., Puls, J., Corral, L. J., \& Villamariz, M. R. 2001, A\&A, 366, 623

Howarth, I. D., \& Prinja, R. K. 1989, ApJS, 69, 527

Howarth, I. D., Siebert, K. W., Hussain, G. A. J., \& Prinja, R. K. 1997, MNRAS, 284, 265

Humphreys, R. H., \& Sandage, A. 1980, ApJS, 44, 319

Ivanov, G. R., Freedman, W. L., \& Madore, B. F. 1993, ApJS, 89,85

Jenkins, E. B. 1970, in IAU Symp. 36 on Ultraviolet Stellar Spectra and Ground Based Observations, ed. L. Houziaux, \& H. E. Butler (Dordrecht: Reidel), 281

Kaper, L., Henrichs, H. F., Nichols, J. S., \& Telting, J. H. 1999, MNRAS, 344, 231

Kudritzki, R. P. 1998, VIII Canary Islands Winter School of Astrophysics on Stellar Astrophysics for the Local Group, ed. A. Aparicio, A. Herrero, \& F. Sanchez (Cambridge University Press), 149

Kudritzki, R. P., Lennon, D. J., \& Puls, J. 1995, Proc. of ESO Workshop Science with the VLT (Heidelberg: Springer), 246

Kudritzki, R. P., \& Puls, J. 2000, ARA\&A, 38, 613

Kudritzki, R. P., Puls, J., Lennon, D. J., et al. 1999, A\&A, 350,970

Lamers, H. J. G. L. M., Haser, S. M., de Koter, A., \& Leitherer, C. 1999, ApJ, 516, 872

Massey, P., Bianchi, L., Hutchings, J., \& Stecher, T. P. 1996, ApJ, 469, 629

McCarthy, J. K., Kudritzki, R. P., Lennon, D. J., Venn, K. A., \& Puls, J. 1997, ApJ, 482, 757

Monteverde, M. I., Herrero, A., \& Lennon, D. J. 2000, ApJ, 545,183

Monteverde, M. I., Herrero, A., Lennon, D. J., \& Kudritzki, R. P. 1996, A\&A, 312, 24

Pauldrach, A. W. A., \& Puls, J. 1990, A\&A, 237, 409

Prinja, R. K. 1990, MNRAS, 246, 392

Prinja, R. K., \& Crowther, P. A. 1998, MNRAS, 300, 828

Puls, J., Springmann, U., \& Lennon, M. 2000, A\&AS, 141, 23

Puls, J., Kudritzki, R. P., Herrero, A., et al. 1996, A\&A, 305, 171

Shull, J. M., \& Van Steenberg, M. E. 1985, ApJ, 294, 599

Vink, J. S., de Koter, A., \& Lamers, H. J. G. L. M. 2000, A\&A, 362,295

Walborn, N. R., Lennon, D. J., Haser, S. M., Kudritzki, R. P., \& Voels, S. A. 1995, PASP, 107, 104 